\title{
Situación de la epilepsia en México y América Latina
}

\section{The situation of epilepsy in Mexico and Latin America}

\author{
Guillermo Noriega-Morales, ${ }^{*}$ Paul Shkurovich-Bialik*,‡
}

Citar como: Noriega-Morales G, Shkurovich-Bialik P. Situación de la epilepsia en México y América Latina. An Med (Mex). 2020; 65 (3): 224-232. https://dx.doi.org/10.35366/95680

\section{RESUMEN}

La epilepsia representa una de las enfermedades crónicas más frecuentes en el mundo que ocasiona altos índices de mortalidad, comorbilidad e invalidez, particularmente en países en desarrollo. Las características clínicas, epidemiológicas y el impacto que ocasiona en quienes la padecen y en su entorno social varían considerablemente en cada región, por lo que es fundamental conocer y entender las peculiaridades de la epilepsia en América Latina y en México. Objetivo: analizar la información más reciente sobre la epidemiología, características e impacto socioeconómico de la epilepsia en México, y compararla con respecto a la epidemiología descrita en América Latina y el mundo. Se realizó una búsqueda bibliográfica de estudios epidemiológicos de prevalencia e incidencia, el efecto en costos, calidad de vida y desgaste de la epilepsia en México, así como revisiones sobre la epidemiología en América Latina y el mundo. Se encontraron seis estudios enfocados en la prevalencia de la epilepsia en México que reportan tasas de 3.9 a 42.2 por 1,000 habitantes así como

\section{ABSTRACT}

Epilepsy represents one of the most frequent chronic diseases in the world, causing high rates of mortality, comorbidity and disability, particularly in developing countries. The clinical and epidemiological characteristics and the impact it causes on those who suffer it and on their social environment vary greatly by region, so it is essential to understand the peculiarities of epilepsy in Latin America and Mexico. Objective: To analyze the most recent information of the epidemiology, characteristics and socioeconomic impact of epilepsy in Mexico, as well as to compare it with respect to the epidemiology described in Latin America and the world. A bibliographic search of epidemiological studies of prevalence and incidence, costs, quality of life and burden of epilepsy in Mexico, as well as reviews of epidemiology in Latin America and the world. Six studies focused on the prevalence of epilepsy in Mexico were found, reporting rates of 3.9 to 42.2 per 1,000 inhabitants, while other studies found high proportions of low quality of life and burden in Mexican patients and their relatives. Prevalence rates

\footnotetext{
* Especialista en Neurología. Departamento de Neurofisiología Clínica.

‡ Especialista en Neurología y Epilepsia. Clínica de Epilepsia.
}

Centro Neurológico. Centro Médico ABC. México.

Recibido para publicación: 03/05/2020. Aceptado: 12/08/2020.

Correspondencia:

Paul Shkurovich-Bialik

Av. Carlos Fernández Graef Núm. 154, Santa Fe, Contadero,

Cuajimalpa de Morelos, 05330, Ciudad de México.

Teléfono: 5511031600

E-mail: paul.shkurovich@gmail.com

Abreviaturas:

$\mathrm{SNC}=$ Sistema nervioso central.
PED = Países en desarrollo

PCE $=$ Pacientes con epilepsia .

$\mathrm{AL}=$ América Latina .

$\mathrm{GBD}=$ Global Burden of Disease Study.

$E A=$ Epilepsia activa.

$\mathrm{PEE}=$ Prevalencia estandarizada por edad .

MEE = Mortalidad estandarizada por edad.

DALY = Años de vida de discapacidad ajustados.

NCC $=$ Neurocisticercosis.

$\mathrm{EVC}=$ Evento vascular cerebral

$\mathrm{CD}=$ Costos directos .

$\mathrm{PD}=$ Países desarrollados.

$\mathrm{EFR}=$ Epilepsia farmacorresistente.

$\mathrm{CV}=$ Calidad de vida .

$\mathrm{ND}=$ Nivel de desgaste .

LTE $=$ Epilepsia de por vida . 
estudios con proporciones elevadas de baja calidad de vida y desgaste en pacientes y familiares mexicanos. Las tasas de prevalencia son similares a las encontradas en América Latina y superiores a las de países desarrollados. La epilepsia presenta mayor prevalencia en países en desarrollo y en México. Aunque la mayoría de los pacientes alcanzan un buen control, la brecha terapéutica es amplia. Su impacto en nuestro país es alto económica y socialmente, lo que enfatiza la importancia de su estudio y manejo.

Palabras clave: Epilepsia, epidemiología, América Latina, México.

Nivel de evidencia: III

\section{INTRODUCCIÓN}

La epilepsia es una enfermedad crónica del sistema nervioso central (SNC) que afecta a individuos de todas las edades, con una distribución mundial. Se caracteriza por una predisposición persistente a generar crisis epilépticas producidas por actividad neuronal cerebral anormal, excesiva y sincronizada. ${ }^{1-4}$ El diagnóstico que podría no ser una tarea complicada, se dificulta particularmente en países en desarrollo (PED), donde existen obstáculos culturales y socioeconómicos para la aceptación y diagnóstico de la enfermedad. ${ }^{1,5}$ La prevalencia de la epilepsia varía entre países, y es más alta en PED, donde se estima se podría encontrar hasta $80 \%$ de los pacientes con epilepsia (PCE), particularmente en las regiones de África subsahariana y América Latina (AL) ${ }^{6}$ Las diferencias metodológicas en los estudios de prevalencia a través del mundo no parecen explicar esta variación. La distribución de algunos factores de riesgo o factores etiológicos como enfermedades infecciosas, traumatismos craneales, problemas perinatales y obstétricos, y factores genéticos podrían explicar algunas de estas diferencias. ${ }^{7}$ En México se han realizado múltiples estudios para valorar la prevalencia de la epilepsia, la mayoría muestran cifras mayores que las estimadas a nivel mundial y con gran variabilidad en las estimaciones entre ellas; las diferencias en sus resultados podrían relacionarse a cambios metodológicos y de la población que han sucedido a través del tiempo. ${ }^{8-13}$ Debido a su prevalencia la epilepsia es una de las enfermedades neurológicas más importantes del mundo; la recurrencia de crisis así como sus consecuencias físicas y psicológicas la hacen una enfermedad desgastante y devastadora para quien la padece y su entorno familiar y social. ${ }^{1,5}$

El objetivo de este estudio fue realizar un análisis de la información más reciente sobre la epidemiolo- are similar to those found in Latin America and higher than in those in developed countries. Epilepsy has a higher prevalence in developing countries and in Mexico. Although most of the patients achieve good control, the therapeutic gap is wide. Its impact in our country is high economically and socially, manifesting the importance of its study and management.

Keywords: Epilepsy, epidemiology, Latin America, Mexico.

Level of evidence: III

gía, características e impacto socioeconómico de la epilepsia en México así como comparar los hallazgos con respecto a la epidemiología descrita en América Latina y el mundo.

\section{MATERIAL Y MÉTODOS}

Se realizó una búsqueda de artículos entre abril y mayo de 2020 en MEDLINE, EMBASE, Medigraphic y SciELO, incluyendo las palabras clave: «epilepsia», «epidemiología», «prevalencia», «incidencia», «México», «América Latina», «costos», «social», «socioeconómico», «calidad de vida», «desgaste», y sus equivalentes en el idioma inglés. Se incluyeron todos los artículos publicados en español o inglés que reportaran: epidemiología descriptiva que abordara el cálculo de tasas de prevalencia, incidencia o ambas, el efecto en costos, calidad de vida y desgaste de la epilepsia en México, así como revisiones sobre la epidemiología en América Latina y el mundo para realizar su comparación. Se incluyeron también los trabajos referenciados en los artículos revisados cuando éstos cumplieron los criterios. Se eliminaron aquellos artículos a los que no se tuvo acceso al texto completo y los que contaran con información duplicada. Los estudios fueron revisados por ambos autores.

Los artículos se clasificaron por región de procedencia, año de publicación, entre estudios de base poblacional (con metodología «de puerta en puerta» o los diseñados con estrategia en dos o tres fases) y estudios basados en registros sanitarios, según el tipo de población analizada (urbana o rural) y el tipo de pacientes evaluados (niños y/o adultos).

Tras la revisión de los resúmenes de los artículos encontrados en las distintas bases de datos, se incluyeron seis estudios enfocados en la epidemiología de prevalencia e incidencia de la epilepsia en México que cumplieron los criterios, y el resto sobre sus ca- 
racterísticas e impacto socioeconómico. Se incluyeron artículos de revisión relevantes en América Latina y el mundo para establecer la comparación con los hallazgos en México.

\section{Epidemiología de la epilepsia en el mundo y América Latina}

De acuerdo con el análisis sobre la carga de la epilepsia a nivel mundial del Global Burden of Disease Stu$d y$ (GBD) publicado en 2019, basado en publicaciones de 195 países entre 1990 y 2016, se estimó la cifra de 45.9 millones de individuos con epilepsia activa (EA) en el mundo en 2016, con una prevalencia estandarizada por edad (PEE) de 6.21 por 1,000 habitantes; observándose mayor PEE en las regiones de África, Asia Central, AL andina y Centroamérica (incluyendo México). El estudio registró 126,055 muertes por epilepsia, la epilepsia idiopática representó $0.23 \%$ de las muertes por cualquier causa en el mundo, con una tasa de mortalidad estandarizada por edad (MEE) de 1.74 por 100,000 habitantes. La epilepsia produjo 13.5 millones de DALYs perdidos en el mundo («años de vida de discapacidad ajustados»: años de vida perdidos, más años con discapacidad por enfermedad) que para la epilepsia idiopática representó 1.82 DALYs perdidos por 1,000 habitantes estandarizados por edad. Se observó un pico de prevalencia de la epilepsia en las edades de cinco a nueve años y mayores de 80 años, sin diferencias entre hombres y mujeres. Al realizarse un análisis entre los datos encontrados entre 1990 y 2016, no se observó un cambio en la PEE mundial, aunque sí una reducción en la MEE de la epilepsia idiopática en 74 países (México uno de ellos), asociada a una reducción de los DALYs perdidos. Un hallazgo relevante del estudio fue una mayor PEE de formas de epilepsia con menor control en PED. ${ }^{5}$ Análisis realizados sobre el GBD en 2017 han mostrado que de los países norteamericanos, México posee una mayor proporción de pérdida de DALYs por epilepsia. ${ }^{14,15}$ Un metaanálisis efectuado en 2017 con base en 222 estudios que igualmente evaluó la epidemiología mundial de la epilepsia, encontró datos similares a los reportados previamente: una prevalencia estimada de EA de 6.38 por 1,000 habitantes (una prevalencia estimada de epilepsia de por vida (LTE) de 7.60 por 1,000 habitantes) y una incidencia anual de 0.614 por 1,000 habitantes/año. De la misma forma, se observó mayor prevalencia e incidencia de epilepsia en PED, particularmente en AL, África y el Medio Oeste. Aunque el análisis mostró resultados heterogéneos entre los estudios evaluados, se consideró que estas diferencias podrían producirse por una mayor presencia de infecciones de SNC, consanguinidad, historia familiar de epilepsia y factores de riesgo perinatal/prenatal en PED. ${ }^{16}$

Un estudio de prevalencia enfocado en AL publicado en 2005 analizó 35 estudios de 11 países, encontrando una prevalencia de EA de 12.4 por 1,000 habitantes y una prevalencia media de epilepsia de por vida de 17.8 por 1,000 habitantes (rango de 6.0 a 44.3) así como una incidencia de 0.77 a 1.90 por 1,000 habitantes en AL. Se observó una gran variabilidad de prevalencia entre los países estudiados, posiblemente asociada con variaciones metodológicas en los estudios, diferencias socioeconómicas y de los sistemas de salud de cada país (Figura 1). ${ }^{17}$ Un metaanálisis de estudios de prevalencia en AL mostró hallazgos similares: una prevalencia de EA de 10.7 por 1,000 habitantes y una prevalencia media de epilepsia de por vida de 15.8 por 1,000 habitantes así como la incidencia de 1.38 por 1,000 habitantes. Este estudio reveló tasas de prevalencia de EA y prevalencia media de epilepsia de por vida más altas en PED, 14 y 18.6 por 1,000 habitantes respectivamente. ${ }^{18}$ Otro estudio sobre la prevalencia de EA en $\mathrm{AL}$ y España evidenció una relación inversa entre el PIB



Figura 1: Prevalencias de epilepsia de por vida en distintos países de América Latina obtenidas de estudios comunitarios (rangos mencionados cuando había más de un estudio por país). Adaptado de: Burneo JG, Tellez-Zenteno J, Wiebe S. Understanding the burden of epilepsy in Latin America: a systematic review of its prevalence and incidence. Epilepsy Res. 2005; 66: 63-74..$^{17}$ 

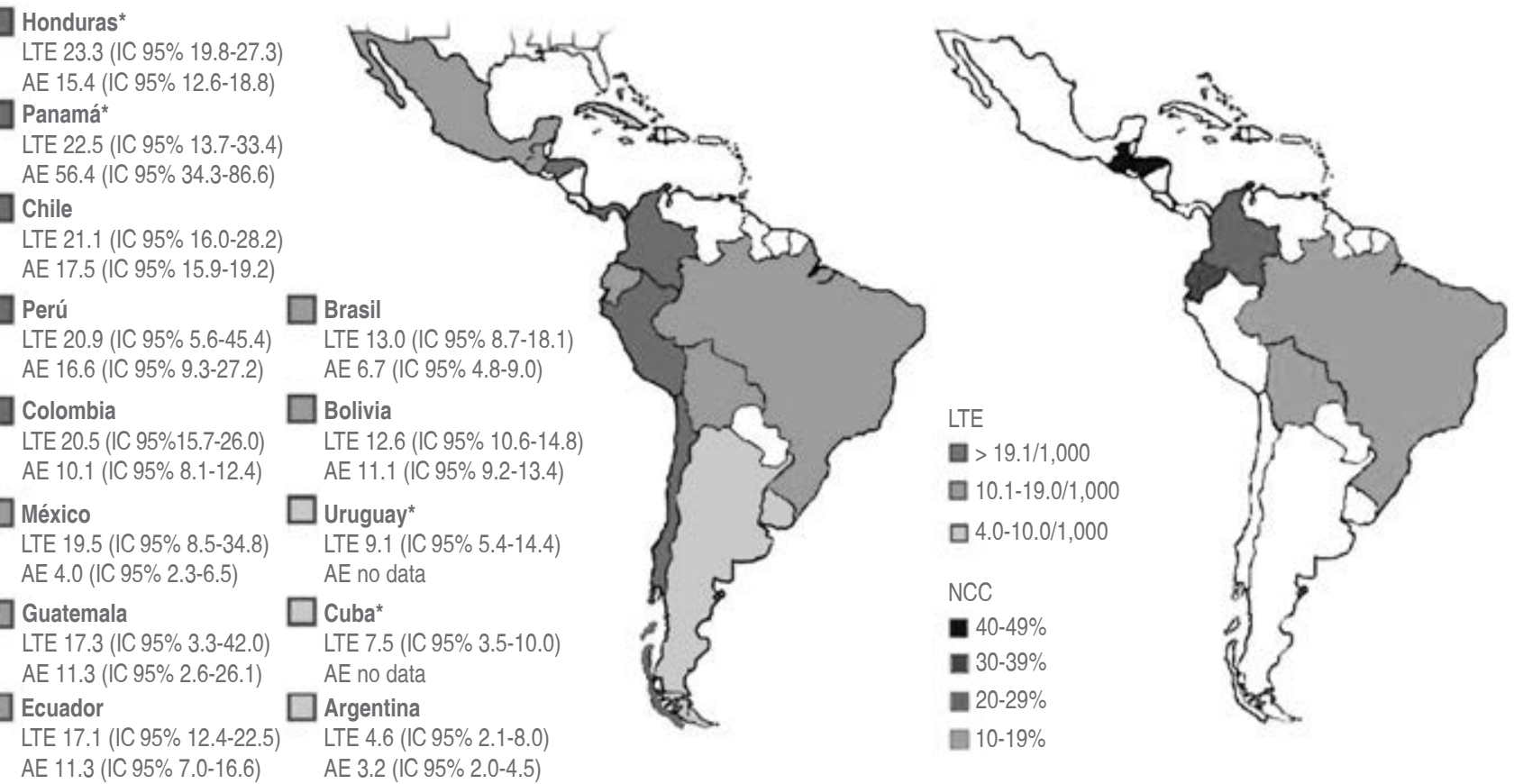

Figura 2: Prevalencia combinada de epilepsia de por vida (LTE) y epilepsia activa (AE) y prevalencia de neurocisticercosis (NCC por tomografía computarizada) e intervalos de confianza al 95\% por 1,000 habitantes en países de América Latina. Adaptado de: Bruno E, Bartoloni A, Zammarchi L, Strohmeyer M, Bartalesi F, García HH et al. Epilepsy and neurocysticercosis in Latin America: a systematic review and meta-analysis. PLoS Negl Trop Dis. 2013; 7 (10): e2480. ${ }^{18}$

* Estimados obtenidos de un solo estudio.

per capita y la prevalencia de EA. ${ }^{19}$ Entre los estudios que realizaron análisis de publicaciones sobre prevalencia combinada de EA y de epilepsia de por vida en $\mathrm{AL},{ }^{18}$ los países que mostraron mayor y menor prevalencia en orden descendente fueron: los de mayor prevalencia Honduras, ${ }^{20}$ Panamá, ${ }^{21,22}$ Chile, ${ }^{23,24}$ Perú ${ }^{25,26}$ y Colombia; ${ }^{27,28}$ y de menor prevalencia Uruguay, ${ }^{29} \mathrm{Cuba}^{30}$ y Argentina. ${ }^{31,32}$ México se encuentra en un rango intermedio (Figura 2).

Entre las infecciones del SNC, la neurocisticercosis (NCC) es considerada una de las principales causas de epilepsia adquirida en PED. Un metaanálisis y revisión sistemática publicado en 2013 sobre población de AL valoró el efecto de la neurocisticercosis en la prevalencia e incidencia de epilepsia. Se calculó una proporción estimada de pacientes con NCC y epilepsia en AL de $32.3 \%$, con mayor proporción en pacientes en áreas rurales $(37.5 \%)$, habiendo una asociación de mayor proporción en población de NCC con mayor prevalencia de EA o de epilepsia de por vida. Adicionalmente este estudio describió una brecha terapéutica estimada de $60.6 \%$ en todos los países de AL, con mayor proporción en regiones rurales (77.8\%). Estos hallazgos refuerzan algunas hipótesis planteadas sobre las causas de mayor prevalencia de epilepsia en AL. ${ }^{18}$

\section{Epidemiología de la epilepsia en México}

En nuestro país se han realizado múltiples estudios para valorar la prevalencia de epilepsia, algunos en las décadas de los años 70 y 80 y otros en las últimas dos décadas son particularmente relevantes (Tabla 1). Los estudios de Gutiérrez-Ávila y colaboradores y de García-Pedroza y su equipo emplearon metodología similar, y fueron publicados en 1980 y 1983 respectivamente: ${ }^{9,10}$ ambos estudios contemplaban una fase de captación de pacientes en la comunidad y otra de corroboración del diagnóstico por un neurólogo y estudios paraclínicos. De éstos, el primer estudio fue llevado a cabo en la población rural de Tecomatlán y realizado «de puerta en puerta» estudiando niños de seis a 12 años, mismo que reveló una prevalencia de epilepsia entre 25 y 41 por 1,000 habitantes; ${ }^{9}$ el segundo estudio fue realizado en 70 escuelas primarias de una población urbana (Tlalpan) en niños de nueve años, con captación mediante cuestionarios a jefes de familia, el cual detectó una prevalencia estimada de epilepsia promedio de 42.2 por 1,000 habitantes, ${ }^{10}$ similar a la hallada en el estudio previo. El tercer estudio relevante de esa época, realizado «de puerta en puerta» y publicado en 1979, analizó la totalidad 
de la población rural del municipio Luvianos, con un interrogatorio estandarizado. Un neurólogo y estudios de gabinete corroboraron el diagnóstico, se halló una tasa de prevalencia de 5.8 por 1,000 habitantes, tasa más baja que la reportada por Gutiérrez-Ávila y García-Pedroza. De estos PCE, 54\% se encontraban entre 10 y 24 años de edad, y la mayoría inició la enfermedad entre 0 y nueve años. ${ }^{8}$

Los tres estudios más recientes se efectuaron «de puerta en puerta», dos en población rural y uno en población urbana. El primero realizado en ciudad Tepatitlán y publicado en 2002 obtuvo información de $5.3 \%$ de la población mediante un cuestionario y corroboró el diagnóstico con un médico neurólogo, se observó una prevalencia de epilepsia de 6.8 casos por 1,000 habitantes, siendo el grupo con mayor proporción de pacientes el de menores de 14 años. ${ }^{11} \mathrm{El}$ segundo estudio publicado en 2011 se realizó en la comunidad rural de San Andrés Azumiatla mediante encuestas a domicilio a $84.5 \%$ de las familias y posteriormente neurólogos corroboraron el diagnóstico en una valoración. Se encontró una prevalencia de 3.9 casos por 1,000 habitantes, siendo el grupo con mayor proporción de pacientes los menores de 40 años. ${ }^{12}$ El tercer estudio publicado en 2015 fue realizado en la comunidad rural de Xocotitla mediante interrogatorio en la totalidad de las casas de la comunidad. De los 33 habitantes identificados con historia de crisis epilépticas $66 \%$ fueron clasificados con epilepsia. La prevalencia de epilepsia y crisis epiléptica sin recu- rrencia se calculó en 25.4 por 1,000 y 38.2 por 1,000 habitantes respectivamente. $57 \%$ de los pacientes tuvieron su primera crisis antes de los 10 años. ${ }^{13}$ Los primeros dos estudios revelaron una proporción de crisis generalizadas de 48 a $75 \%$ y parciales de 25 a $52 \%$. Estos últimos tres estudios más recientes muestran en general tasas de prevalencia más bajas que en el periodo anterior. La disparidad en las prevalencias entre los estudios comentados puede deberse a distintos motivos: tipo de población evaluada (rural o urbana), individuos evaluados (niños y/o adultos; considerando que uno de los picos de incidencia se encuentra en la infancia), diferencias metodológicas en el diagnóstico de epilepsia (criterios empleados), y la probabilidad de un cambio real en la incidencia a través del tiempo.

Para caracterizar la epilepsia en niños dos estudios descriptivos realizados en ambiente hospitalario han sido relevantes: un estudio publicado en 2002 por Ruiz-García y colaboradores y llevado a cabo en el Instituto Nacional de Pediatría en 719 pacientes menores de 18 años con epilepsia (57\% hombres), con seguimiento promedio de cinco años; ${ }^{33}$ y otro estudio realizado en el Hospital General de Zona No. 47 del Instituto Mexicano del Seguro Social en 200 pacientes menores de 16 años con epilepsia (55\% hombres), con seguimiento de un año promedio y publicado en 2004 por Serrano-Martín. ${ }^{34}$ Estos estudios mostraron una proporción de epilepsia idiopática de 17 a $26 \%$, sintomática de 64 a $74 \%$ y crip-

Tabla 1: Estudios de prevalencia de epilepsia realizados en México.

\begin{tabular}{|c|c|c|c|c|c|c|c|}
\hline Estudio & Tipo de estudio & Localidad & $\begin{array}{l}\text { Tipo de } \\
\text { población }\end{array}$ & $\begin{array}{l}\text { Grupo } \\
\text { evaluado }\end{array}$ & $\begin{array}{c}\text { Población } \\
\text { total/evaluada }\end{array}$ & $\begin{array}{l}\text { Tipo de } \\
\text { evaluación }\end{array}$ & $\begin{array}{l}\text { Prevalencia estimada } \\
\text { (por cada } 1,000 \\
\text { habitantes) }\end{array}$ \\
\hline $\begin{array}{l}\text { Márquez } \\
(1979)^{8}\end{array}$ & $\begin{array}{l}\text { «de puerta en } \\
\text { puerta» }\end{array}$ & $\begin{array}{l}\text { Luvianos/Estado de } \\
\text { México }\end{array}$ & Rural & $\begin{array}{l}\text { Cualquier } \\
\text { edad }\end{array}$ & $4,103 / 4,103$ & $Q+N+G$ & 5.8 \\
\hline $\begin{array}{l}\text { Gutiérrez-Ávila } \\
(1980)^{9}\end{array}$ & $\begin{array}{c}\text { «de puerta en } \\
\text { puerta» }\end{array}$ & $\begin{array}{l}\text { San Miguel Tecomatlán/ } \\
\text { Estado de México }\end{array}$ & Rural & $\begin{array}{c}\text { Niños de } 6 \text { a } \\
12 \text { años }\end{array}$ & $360 / 360$ & $Q+N+G$ & $25-41$ \\
\hline $\begin{array}{l}\text { Cruz-Alcalá } \\
(2002)^{11}\end{array}$ & $\begin{array}{l}\text { "de puerta en } \\
\text { puerta» }\end{array}$ & $\begin{array}{c}\text { Ciudad Tepatitlán/ } \\
\text { Jalisco }\end{array}$ & Urbana & $\begin{array}{l}\text { Cualquier } \\
\text { edad }\end{array}$ & $170,000 / 9,082^{*}$ & $Q+N$ & 6.8 \\
\hline $\begin{array}{l}\text { Quet } \\
(2011)^{12}\end{array}$ & $\begin{array}{c}\text { "de puerta en } \\
\text { puerta» }\end{array}$ & $\begin{array}{c}\text { San Andrés Azumiatla/ } \\
\text { Puebla }\end{array}$ & Rural & $\begin{array}{l}\text { Cualquier } \\
\text { edad }\end{array}$ & $6,203 / 4,008$ & $Q+N$ & 3.9 \\
\hline
\end{tabular}

$\mathrm{Q}=$ cuestionario, $\mathrm{N}$ = diagnóstico corroborado por neurólogo, $\mathrm{G}$ = estudio de electroencefalograma y/o imagen.

* Población estudiada determinada mediante cálculo de muestra. 
togénica de $18 \%$, con edades promedio de 10 , cinco y siete años respectivamente para cada grupo. Los síndromes epilépticos más frecuentes en las formas idiopáticas fueron: epilepsia idiopática con crisis tónico-clónicas generalizadas de 45 a 53\%, epilepsia con crisis de ausencia $25 \%$, epilepsia rolándica benigna $12 \%$. En la epilepsia criptogénica el síndrome de West representó de 5 a $7 \%$ y Lennox-Gastaut de 3 a 13\%. De los PCE sintomática, las principales causas precipitantes fueron: encefalopatía anoxo-isquémica de 24 a $49 \%$ (19\% en el periodo perinatal), neuroinfecciones e infecciones parasitarias de 10 a $21 \%$, evento vascular cerebral (EVC) $16 \%$, traumatismo $12 \%$, enfermedad neurocutánea $7 \%$ y disgenesias cerebrales de 3 a 6\%. En el primer estudio 65\% de los pacientes en cada grupo de clasificación de epilepsia tenían remisión de crisis con un solo fármaco, siendo el valproato el fármaco más utilizado seguido de carbamazepina y fenitoína. En el segundo estudio $68 \%$ tenía un solo fármaco y $75 \%$ de todos los pacientes se encontraban libres de crisis.

Un estudio de casos y controles realizado por Cruz-Cruz y colaboradores en el Hospital General Regional No. 1 «Vicente Guerrero» en Acapulco ha analizado posibles factores de riesgo de epilepsia en niños en México. El estudio seleccionó pacientes de 28 días de edad a 15 años (118 casos y 118 controles). Las características de la población mostraron una proporción de 55 y $56 \%$ hombres en grupo de casos y controles respectivamente; $35 \%$ de los pacientes eran menores de cinco años, 40\% tenían de seis a 10 años y $25 \%$ de 11 a 15 años; $66 \%$ de los pacientes se clasificaron con epilepsia idiopática y $34 \%$ con epilepsia sintomática. El estudio halló una diferencia estadísticamente significativa en la proporción de: antecedentes familiares de epilepsia en primer grado ( 20 vs. $10 \%$ en casos y controles respectivamente), infección de vías urinarias durante la gestación (33 vs. $22 \%$ ), asfixia al nacimiento (28 vs. $15 \%$ ), sugiriendo estos rasgos como posibles factores de riesgo de desarrollar epilepsia. ${ }^{35}$

Un estudio prospectivo observacional publicado en 2009 que evaluó 455 casos de epilepsia de pacientes entre 20 y 89 años en 10 centros neurológicos en diferentes estados de la República y la capital, nos ha permitido caracterizar la epilepsia de adultos en México. En éste los grupos etarios más grandes fueron el de 20 a 30 años con $29 \%$ de los pacientes y el de 31 a 40 años con $23 \%$ de los pacientes, y $50.5 \%$ de los pacientes fueron hombres. La distribución del tipo de crisis mostró que $49 \%$ de los pacientes presentaba crisis generalizadas, y $51 \%$ crisis focales (de éstas $32 \%$ complejas, $26 \%$ motoras simples y $24 \%$ simples con generalización secundaria). Con respecto al tipo de epilepsia $48 \%$ se clasificó con epilepsia criptogénica, y el resto con epilepsia sintomática, siendo las etiologías más frecuentes: NCC en $21 \%$, EVC en $17 \%$ y neoplasias en $5 \%$. Todos los pacientes se encontraban con tratamiento antiepiléptico, 83\% en monoterapia (los fármacos más empleados: fenitoína 50\%, carbamazepina en $31 \%$, valproato $7 \%) ; 60 \%$ de los pacientes presentaban una frecuencia de crisis de una al mes, $6 \%$ más de 10 crisis al mes y el resto un rango intermedio. ${ }^{36}$ En un estudio sobre los motivos de descontrol, motivo de visita a urgencias en PCE adultos se observó que la falta de adhesión a tratamiento produjo $27 \%$ de éstas (incluyendo olvido de dosis, negativa a tomarlo, causas económicas e indicaciones contradictorias entre médicos), seguida de causas no determinadas, infecciones y privación de sueño. ${ }^{37}$

\section{Consecuencias sociales y económicas de la epilepsia en México}

La epilepsia es una de las enfermedades neurológicas más frecuentes del mundo, y aunque es una enfermedad tratable, usualmente requiere de tratamiento de por vida y en ocasiones manejo quirúrgico. Los altos costos de su atención se relacionan con su estudio, tratamiento, hospitalizaciones así como pérdida de empleo, ingresos y trabajo doméstico. En una revisión sistemática de estudios internacionales se han calculado los costos directos (CD) del sistema de salud anuales por paciente en países desarrollados (PD) de 1,736 a 2,813 dólares (2,384 dólares en el primer año de diagnóstico) y de 5,848 dólares anuales en pacientes que consultan por cirugía de epilepsia; la mayor parte de los costos comprende los fármacos antiepilépticos y los servicios hospitalarios. Los costos varían según la gravedad de la epilepsia y respuesta a tratamiento, siendo más altos en pacientes en protocolo quirúrgico y epilepsia farmacorresistente (EFR) ${ }^{38}$ En México, donde previamente se ha mostrado un retraso promedio de 14.1 años entre el inicio de crisis y cirugía de epilepsia, ${ }^{39}$ un estudio valoró en 2006 los CD de la atención de EFR en pacientes del Instituto Mexicano del Seguro Social, calculando costos anuales por paciente de 2,646 dólares (3,483 dólares en quienes requirieron de hospitalización). Extrapolando estos costos a un posible número de pacientes con EFR que pudiera atender la institución a nivel nacional, los autores estimaron un costo anual de 232 millones de dólares. ${ }^{40}$ En Estados 
An Med (Mex) 2020; 65 (3): 224-232

Unidos un estudio observó una reducción en los CD entre 6,806 y 13,454 dólares anuales en PCE posterior a tratamiento quirúrgico con respecto a pacientes pareados, también menos citas médicas y citas a urgencias/hospitalización, ${ }^{41}$ resaltando la importancia económica de esta medida terapéutica.

El análisis de "gastos de bolsillo" (pagos directos a proveedores de servicios de salud realizados por los individuos al momento de su uso) en PD ha mostrado costos de 9.3 a 196 dólares por mes por paciente, y costos por hospitalización de 1,018 dólares en pacientes no asegurados. En PED el "gasto de bolsillo» puede representar hasta $20 \%$ del ingreso familiar; lo que a su vez se ha asociado a mal apego a tratamiento. Adicionalmente se han calculado costos anuales por paciente de 2,037 a 8,586 dólares por "pérdida de productividad» en $\mathrm{PD}$, ya sea por pérdida de trabajo del paciente o del cuidador. ${ }^{38}$ En pacientes con epilepsia de lóbulo temporal con resistencia a fármacos, la cirugía ha sido un factor asociado con menor desempleo, ${ }^{38}$ nuevamente enfatizando su relevancia. En México un estudio realizado en 56 PCE en edad laboral reveló que $32 \%$ se encontraban desempleados y $29 \%$ nunca habían laborado, de éstos, $44 \%$ relacionaron el desempleo con no poder laborar por discapacidad y $25 \%$ refirieron no haber sido empleados por las empresas por discapacidad física; incluso algunos pacientes reportaron despidos asociados a discriminación por epilepsia. ${ }^{42}$ Este fenómeno fue observado en otro estudio mexicano de 10 pacientes con epilepsia de lóbulo temporal, los cuales expresaron dificultades para hallar o mantener trabajos por discriminación, y de los cuales sólo dos se encontraban empleados; además la mayoría refirió haber sufrido previamente discriminación que limitó su educación. ${ }^{43}$ Considerando estos problemas educativos, otro estudio en 143 niños en edad escolar en población rural y urbana en México reveló que 42\% de ellos fueron rechazados en su ingreso a la escuela ${ }^{44}$ acentuando este ciclo la falta de educación y el desempleo.

Dos estudios realizados en México analizaron el impacto de la epilepsia en la calidad de vida (CV) en PCE, ambos llevados a cabo en ámbito hospitalario y empleando el cuestionario QOLIE-31 (puntuación de 0 a 100, con peor CV con menor puntaje). El primero publicado en 2007 reveló que 50\% de los PCE reportaban una mala CV (puntuación < 60), 31\% regular y el resto de buena a excelente $\mathrm{CV}^{45}$ El segundo publicado en 2005 mostró una calificación promedio de QOLIE-31 para los PCE de 55 puntos y detectó que los trastornos del sueño, un estado socioeconómico bajo, el género femenino y un mal control de crisis se asociaron a reducción de la $\mathrm{CV}^{46}$ Otro estudio en población mexicana de PCE adultos de casos y controles reveló una relación en pérdida de CV por QOLIE-31 y la presencia de depresión/ansiedad, y mala calidad de sueño ${ }^{47}$ acentuado por el conocimiento previo de prevalencias altas de trastornos de ansiedad y depresión (hasta 25\%) en PCE adultos mexicanos. ${ }^{48} \mathrm{La}$ CV también se ve afectada en los cuidadores de PCE mexicanos, lo cual fue demostrado en un estudio realizado en un centro hospitalario de tercer nivel, donde se encontró un nivel de desgaste (ND) moderado en los cuidadores y una calidad de vida con puntajes de 47-55 en la escala WHOQOL-BREF (puntuación de 0 a 100, con peor CV con menor puntaje). Además, se detectó que los cuidadores de PCE con trastornos neuropsiquiátricos (particularmente con agresividad), con menor edad de inicio o mayor tiempo de evolución de epilepsia tenían niveles menores de CV y de mayor ND. ${ }^{49}$ Lo antes expuesto podría asociarse a los hallazgos de otro estudio mexicano realizado en 47 PCE adultos, en el que 34\% de éstos presentó algún tipo de violencia por parte de sus cuidadores, aunque también hubo en $28 \%$ de los cuidadores violencia proveniente de sus PCE. ${ }^{50}$

\section{CONCLUSIONES}

La epilepsia representa uno de los trastornos neurológicos más frecuentes en población pediátrica y adulta; a pesar de que afecta a ambos sexos, estudios epidemiológicos practicados en nuestro país confirman mayor prevalencia en hombres, especialmente cuando las crisis inician antes de los 18 años.

La mayoría de los pacientes pueden alcanzar un buen control de crisis cuando el diagnóstico es oportuno y preciso, y el tratamiento es correctamente seleccionado y dosificado para el tipo de epilepsia o síndrome epiléptico; sin embargo, de los cuatro millones de PCE en AL, se estima que por lo menos tres millones no reciben el tratamiento adecuado; este fenómeno denominado la brecha terapéutica en epilepsia es mayor en los PED que en países con mayor acceso a recursos diagnósticos y terapéuticos.

La etiología de la epilepsia en PD corresponde frecuentemente a malformaciones del desarrollo cortical, patología cerebrovascular y a tumores cerebrales, mientras que en México y en la mayoría de países de AL está asociada con traumatismos craneoencefálicos, infecciones del sistema nervioso central, complicaciones prenatales y perinatales e infestaciones parasitarias por NCC, muchas de ellas causas prevenibles, y resultado de la pobreza y de la falta de educación. 
El impacto que tiene esta enfermedad en nuestro país y otros PED presenta varios matices: los altos costos que produce en nuestras instituciones, los pacientes y sus familias; los problemas laborales que se generan mediante la enfermedad y procesos de discriminación; la reducción en CV en el paciente y sus cuidadores y el desgaste en éstos; la estigmatización en quien la padece y su entorno familiar y social que ocasiona prejuicios. Procesos todos éstos de desgaste que podrían ser menguados a través de una mejor educación, y de un diagnóstico y tratamiento óptimos.

\section{BIBLIOGRAFÍA}

1. Devinsky O, Vezzani A, O'Brien TJ, Jette N, Scheffer IE, de Curtis M et al. Epilepsy. Nat Rev Dis Prim. 2018; 3: 18024.

2. Fisher RS, Acevedo C, Arzimanoglou A, Bogacz A, Cross JH, Christian E et al. ILAE official report: a practical clinical definition of epilepsy. Epilepsia. 2014; 55 (4): 475-482.

3. Scheffer IE, Berkovic S, Capovilla G, Connolly MB, French J, Guilhoto L et al. ILAE classification of the epilepsies: position paper of the ILAE Commission for Classification and Terminology. Epilepsia. 2017; 58 (4): 512-521.

4. Fisher RS, Cross JH, French JA, Higurashi N, Peltola J, Roulet $\mathrm{E}$ et al. Operational classification of seizure types by the International League Against Epilepsy: position paper of the ILAE Commission for Classification and Terminology. Epilepsia. 2017; 58 (4): 522-530.

5. GBD 2016 Epilepsy Collaborators. Global, regional, and national burden of epilepsy, 1990-2016: a systematic analysis for the Global Burden of Disease Study 2016. Lancet Neurol. 2019; 18 (4): 357-375.

6. World Health Organization. Epilepsy. Fact sheet. No. 999. 2012. Updated February 2016. Available in: http://www.who. $\mathrm{int} /$ mediacentre/factsheets/fs999/en/

7. Yemadje LP, Houinato D, Quet F, Druet-Cabanac M, Preux PM. Understanding the differences in prevalence of epilepsy in tropical regions. Epilepsia. 2011; 52 (8): 1376-1381.

8. Márquez L, Olivares L. Epilepsia en México: estudio epidemiológico de una comunidad rural. Salud Publica Mex. 1979; 21 (5): 487-495.

9. Gutiérrez Ávila JH, cols. Prevalencia de epilepsia infantil en una población rural de México. Salud Publica Mex. 1980; 22 (3): 269-273.

10. García-Pedroza F, Rubio-Donnadieu F, García-Ramos G, Escobedo-Ríos F, González-Cortés A. Prevalence of epilepsy in children: Tlalpan, Mexico City, Mexico. Neuroepidemiology. 1983; 2: 16-23.

11. Cruz-Alcalá LE, Vázquez-Castellanos JL. Prevalencia de algunas enfermedades neurológicas en la Ciudad de Tepatitlán, Jalisco, México. Rev Mex Neurocienc. 2002; 3 (2): 71-76.

12. Quet F, Preux PM, Huerta M, Ramirez R, Abad T, Fragoso $\mathrm{G}$ et al. Determining the burden of neurological disorders in populations living in tropical areas: who would be questioned? Lessons from a Mexican rural community. Neuroepidemiology. 2011; 36: 194-203.

13. San-Juan D, Alvarado-León S, Barraza-Díaz J, Davila-Avila NM, Hernandez-Ruíz A, Anschel DJ. Prevalence of epilepsy, beliefs and attitudes in a rural community in Mexico: a doorto-door survey. Epilepsy Behav. 2015; 46: 140-143.

14. Vigo D, Jones L, Thornicroft G, Atun R. Burden of mental, neurological, substance use disorders and self-harm in North
America: a comparative epidemiology of Canada, Mexico, and the United States. Can J Psychiatry. 2020; 65 (2): 87-98.

15. GBD 2017 DALYs and HALE Collaborators. Global, regional, and national disability-adjusted life-years (DALYs) for 359 diseases and injuries and healthy life expectancy (HALE) for 195 countries and territories, 1990-2017: a systematic analysis for the Global Burden of Disease Study 2017. Lancet. 2018; 392 (10159): 1859-1922.

16. Fiest KM, Sauro KM, Wiebe S, Patten SB, Kwon CS, Dykeman $\mathrm{J}$ et al. Prevalence and incidence of epilepsy a systematic review and meta-analysis of international studies. Neurology. 2017; 88: 296-303.

17. Burneo JG, Tellez-Zenteno J, Wiebe S. Understanding the burden of epilepsy in Latin America: a systematic review of its prevalence and incidence. Epilepsy Res. 2005; 66: 63-74.

18. Bruno E, Bartoloni A, Zammarchi L, Strohmeyer M, Bartalesi F, García HH et al. Epilepsy and neurocysticercosis in Latin America: a systematic review and meta-analysis. PLoS Negl Trop Dis. 2013; 7 (10): e2480.

19. García-Martín G, Serrano-Castro PJ. Epidemiología de la epilepsia en España y Latinoamérica. Rev Neurol. 2018; 67 (7): 249-262.

20. Medina MT, Durón RM, Martínez L, Osorio JR, Estrada AL, Zúniga C et al. Prevalence, incidence, and etiology of epilepsies in rural Honduras: the Salamá Study. Epilepsia. 2005; 46 (1): 124-131.

21. Gracia F, de Lao SL, Castillo L, Larreategui M, Archbold C, Brenes MM et al. Epidemiology of epilepsy in Guaymi Indians from Bocas del Toro Province, Republic of Panama. Epilepsia. 1990; 31 (6): 718-723.

22. Gracia FJ, Bayard V, Triana E, Castillo LC, Benzadón A, Larreátegui $\mathrm{M}$ et al. Prevalence of neurologic diseases in Belisario Porras municipality, District of San Miguelito, Panama, 1986. Rev Med Panama. 1988; 13 (1): 40-45.

23. Chiofalo N, Kirschbaum A, Fuentes A, Cordero ML, Madsen J. Prevalence of epilepsy in children of Melipilla, Chile. Epilepsia. 1979; 20 (3): 261-266.

24. Lavados J, Germain L, Morales A, Campero M, Lavados P. A descriptive study of epilepsy in the district of El Salvador, Chile, 1984-1988. Acta Neurol Scand. 1992; 85 (4): 249-256.

25. Montano SM, Villaran MV, Ylquimiche L, Figueroa JJ, Rodriguez S, Bautista CT et al. Neurocysticercosis: association between seizures, serology, and brain CT in rural Peru. Neurology. 2005; 65 (2): 229-233.

26. Trelles L. Neurocysticercosis and epilepsy in Peru. Clin Neurol Neurosurg. 1992; 94 (Suppl): S153-S154.

27. Pradilla G, Vesga B, Leon-Sarmiento F, Bautista L, Nunez L. Neuroepidemiology in the eastern region of Colombia. Rev Neurol. 2002; 34: 1035-1043.

28. Diaz-Cabezas R, Ruano-Restrepo M, Chacon-Cardona J, VeraGonzalez A. Neuroepidemiology profile of the central zone of the department of Caldas (Colombia), years 2004-2005. Rev Neurol. 2006; 43: 646-652.

29. Ketzoian C. Estudio piloto de la prevalencia de las principales enfermedades neurológicas en una población del Uruguay. Rev Med Uruguay. 1992; 8: 191-205.

30. Pascual López MA, Gispert JP, Rivera LR, Ochoa FR, Tejeiros A. Epilepsy: spidemiological study in a child population. Bol Med Hosp Infant Mex. 1980; 37 (4): 811-21.

31. Melcon MO, Kochen S, Vergara RH. Prevalence and clinical features of epilepsy in Argentina. A community-based study. Neuroepidemiology. 2007; 28 (1): 8-15.

32. Somoza MJ, Forlenza RH, Brussino M, Licciardi L. Epidemiological survey of epilepsy in the primary school population in Buenos Aires. Neuroepidemiology. 2005; 25 (2): 62-68. 
33. Ruiz-García M, Sosa-de-Martinez C, González-Astiazarán A, Rueda-Franco F. Clinical-etiological and therapeutic profile of 719 Mexican epileptic children. Childs Nerv Syst. 2002; 18: 593-598.

34. Serrano-Martín J. Aspectos epidemiológicos de la epilepsia en un Hospital General de segundo nivel. Plast Rest Neurol. 2004; 3 (1-2): 39-43.

35. Cruz-Cruz MR, Gallardo-Elías J, Paredes-Solís S, LegorretaSoberanis J, Flores-Moreno M, Andersson N. Factores asociados a epilepsia en niños en México: un estudio casocontrol. Bol Med Hosp Infant Mex. 2017; 74 (5): 334-340.

36. Suástegui R, Gutiérrez J, Ramos R, Bouchan S, Navarrete H, Ruiz J et al. Características clínicas de la epilepsia de inicio tardío en México al principio del nuevo milenio: 455 casos. Rev Investig Clínica. 2009; 61 (5): 354-363.

37. Valdés-Galván RE, González-Calderón G, Castro-Martínez E. Epidemiología del descontrol de la epilepsia en un servicio de urgencias neurológicas. Rev Neurol. 2019; 68 (8): 321-325.

38. Allers K, Essue BM, Hackett ML, Muhunthan J, Anderson CS, Pickles $\mathrm{K}$ et al. The economic impact of epilepsy: a systematic review. BMC Neurol. 2015; 15: 245.

39. Guerrero-Pérez R, Aguado-Carrillo G, Vázquez-Barrón D, Velasco-Campos F, Velasco-Monroy AL. Tiempo estimado entre inicio de crisis y tratamiento quirúrgico en pacientes con epilepsia refractaria. Arch Neurocien. 2014; 19 (3): 153-156.

40. García-Contreras F, Constantino-Casas P, Castro-Ríos A, Nevárez-Sida A, Estrada-Correa G del C, Rivera FC et al. Direct medical costs for partial refractory epilepsy in Mexico. Arch Med Res. 2006; 37: 376-383.

41. Schiltz NK, Kaiboriboon K, Koroukian SM, Singer ME, Love TE. Long-term reduction of health care costs and utilization after epilepsy surgery. Epilepsia. 2016; 57 (2): 316-324.

42. Ponce-Arargo A, Núñez-Orozco L, Medrano-Loera G, ReyesLópez A, Cantú-Quintanilla G. Discriminación laboral en
México a las personas con epilepsia. Repercusión bioética. Rev CONAMED. 2019; 24 (1): 46-52.

43. Espínola-Nadurille M, Crail-Melendez D, Sánchez-Guzmán MA. Stigma experience of people with epilepsy in Mexico and views of health care providers. Epilepsy Behav. 2014; 32: 162-169.

44. Gutiérrez-Moctezuma J, Villegas-Peña H, Solórzano-Gómez E, Hernández-Rodríguez V. El impacto social y educacional por la epilepsia, en niños derechohabientes de tres hospitales de seguridad social. Rev Mex Pediatr. 2000; 67 (3): 111-116.

45. Miranda-Nava G, Martínez-Tenorio FN, Arceo-Guzmán ME. La calidad de vida de los pacientes epilépticos determinada por la aplicación de la escala QOLIE-31. Rev Neurol Neurocir y Psiquiatr. 2007; 40 (2): 50-55.

46. Alanis-Guevara I, Peña E, Corona T, López-Ayala T, LópezMeza E, López-Gómez M. Sleep disturbances, socioeconomic status, and seizure control as main predictors of quality of life in epilepsy. Epilepsy Behav. 2005; 7: 481-485.

47. Camara-Lemarroy CR, Hoyos M, Ibarra-Yruegas BE, DíazTorres MA, De León R. Affective symptoms and determinants of health-related quality of life in Mexican people with epilepsy. Neurol Sci. 2017; 38: 1829-1834.

48. Domínguez-aguilera MC, Muñiz-Landeros CE. Prevalence of psychiatric disorders in patients with epilepsy in a tertiary level care hospital: Detection through the MINI PLUS International Structured Interview. Med Univ. 2017; 19 (74): 4-7.

49. Gutiérrez-Angel AM, Martínez-Juárez IE, HernándezVanegas LE, Crail-Meléndez D. Quality of life and level of burden in primary caregivers of patients with epilepsy: Effect of neuropsychiatric comorbidity. Epilepsy Behav. 2018; 81: 12-17.

50. Sánchez-Guzmán MA, Paz-Rodríguez F, Trujillo-De Los Santos Z, Espinola-Nadurille M. Domestic violence among persons with epilepsy and their caregivers. Epilepsy Behav. 2017; 71: 85-93. 